\section{Journal of Transplantation \& Stem Cell Biology}

\author{
Giuseppe Orlando*, Stephen J. Walker* \\ Wake Forest Institute for Regenerative Medicine, USA \\ Address for Correspondence \\ Giuseppe Orlando, Department of Surgery, Wake Forest Institute for \\ Regenerative Medicine, Medical Center Boulevard, Winston Salem, NC \\ 27157, Tel: +1 336 716-6843; E-mail: gorlando@wakehealth.edu \\ Stephen J. Walker, Wake Forest Health Sciences, Wake Forest Institute \\ for Regenerative Medicine, Medical Center Boulevard, Winston Salem, \\ NC 27157, Tel: +1 336 713-7272; E-mail: swalker@wakehealth.edu \\ Copyright: (c) 2014 Orlando G, et al. This is an open access article \\ distributed under the Creative Commons Attribution License, which \\ permits unrestricted use, distribution, and reproduction in any medium, \\ provided the original work is properly cited. \\ Submission: 02 September 2014 \\ Accepted: 03 September 2014 \\ Published: 05 September 2014
}

Although organ transplantation is now widely viewed as one of the monumental achievements in the history of modern medicine, that was not always the case. Indeed, back at the beginning of the $20^{\text {th }}$ century the idea of replacing diseased organs with new functioning counterparts seemed unthinkable. However, as a result of the vision and perseverance of pioneers, their temerity to challenge ethical and biological paradigms, concomitant progress in surgical techniques and know-how, along with the conception, implementation and development of technologies and the creation of ad hoc infrastructures, the dream eventually became reality and organ transplantation has become routine medical practice. Patients that would have inexorably been destined to death prior to these advances can now have their damaged organs replaced and enjoy a satisfactory quality of life, quite comparable to that of non-transplant individuals.

It took almost an entire century for organ transplantation to become the treatment of choice in a myriad of desperate clinical conditions, and within this history three distinct phases can be identified [1]. The first phase spans from the early 1900s to the introduction of cyclosporine into the clinical practice (FDA approved in 1983), and may be referred to as the surgery era (Figure 1). During this era the surgical technique was conceived and developed, and the foundations of transplant immunology were laid down. Early on, as it became apparent that once organs are pulled out of the body they undergo significant damage, strategies to augment organ preservation were also developed. And, for the first time, surgeons started to imagine that replacement organs may be able to be procured not only from humans, but also from other animal species, and the concept of xenotransplantation was born. However, due to the lack of effective immunosuppressive strategies, unqualified success was not the usual outcome during the surgery era.

The almost accidental discovery of the formidable immunomodulatory properties of cyclosporine, followed by its introduction into clinical practice, revolutionized the field and allowed transplantation to transition from the experimental to the clinical arena, where long-term success then became the typical outcome. This era can be referred to as the immunology era (Figure 1), due to extraordinary progress in the field of transplant immunology and the discovery and introduction into clinical practice of numerous new immunosuppressant drugs. In the early days of this era, surgeons in the field learned how to handle and manage immunosuppression but also realized that immunosuppression exists as a double-edged sword. In fact, the price to pay to allow long term, rejection-free engraftment of the new organ is the onset of a clinical syndrome consisting in the myriad of side effects that come with lifelong immunosuppression and that dramatically impacts on mid- and long-term mortality and morbidity, post-transplant. For this reason, intense research has been and is still being conducted, the primary aim of which is the establishment of an immunosuppression-free status - namely, tolerance. Because the ultimate goal is to provide immunosuppression-free transplantation, tolerance has long been recognized as the Holy Grail of organ transplantation [2]. However, due to the excellent clinical outcomes being achieved even with the need for immunosuppression, the indications for the different types of transplant have multiplied and consequently the demand for organs has increased exponentially, forcing the transplant community to devise and develop new strategies to increase the donor pool. As a result, the disparity between the demand and the supply has increased, followed by a dramatic increase in the mortality and the dropout rate while on the waiting list. Despite numerous strategies that are currently being implemented, the gap between demand and supply remains untenable.

In this the third millennium, transplantation still faces several problems and has many hurdles to overcome and needs that must be met. To address them, current research is focusing on multiple avenues that include: the development and validation of biomarkers of graft dysfunction and immune activation; the identification of novel immune modifiers, like stem cells, new drugs and biologics; the development of new tools to study and/or visualize the human immune response; the identification and validation of surrogate markers for long-term outcomes; the assessment of the effects of cell therapies on protective immunity; the identification of predictors and/or mechanisms of disease after transplant; the application of epigenetics in determining transplant outcomes; the reduction of post-transplant complications; the optimization of organ utilization; the prevention of late graft failure; and the improvement of transplant patient's quality of life.

In the last two decades a new frontier within medical practice, currently referred to as regenerative medicine (labeled as tissue engineering before that), has shown immense potential to meet among others - the most urgent needs of organ transplantation, i.e. 


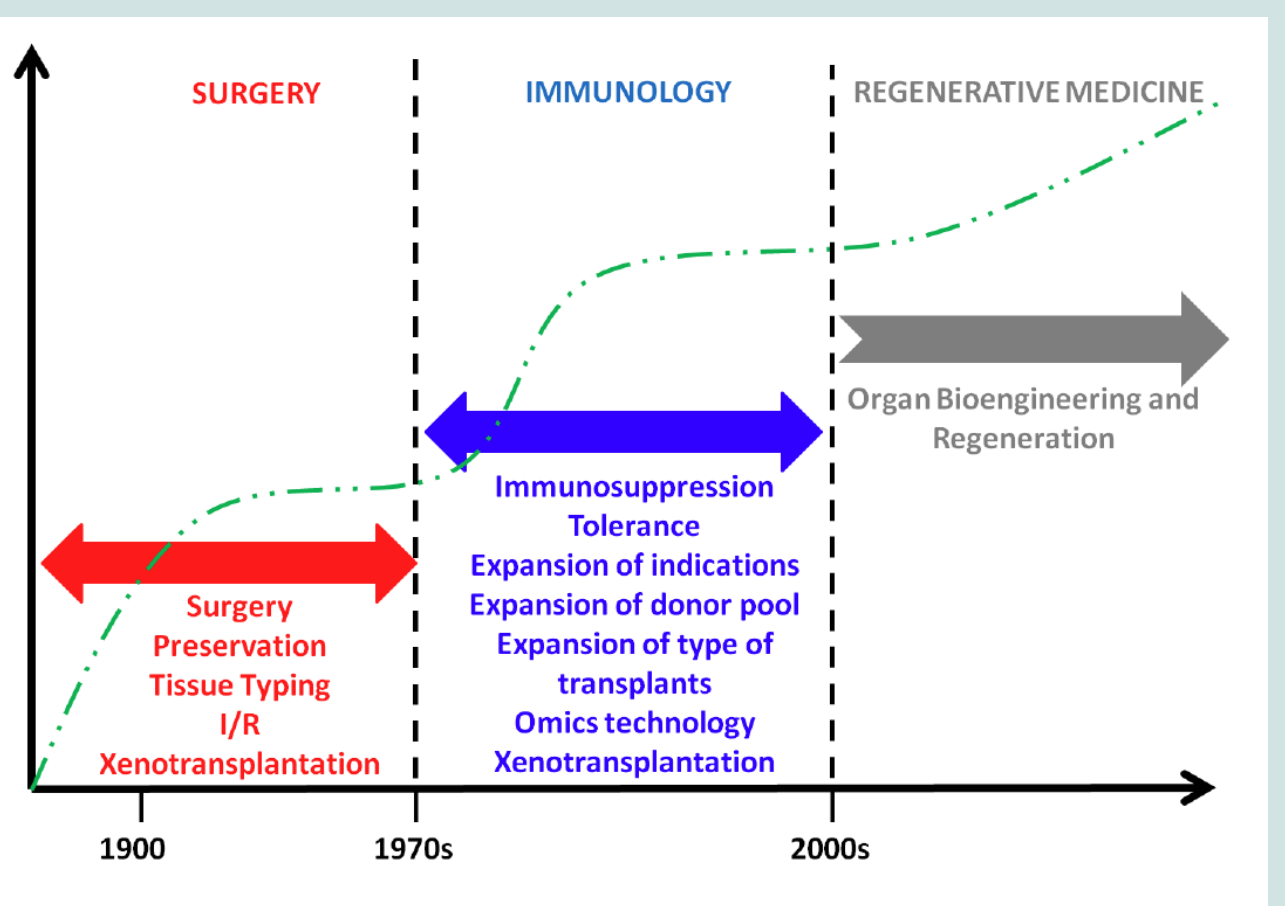

Figure 1: History of transplantation (see text for explanation) (from: Salvatori et al. Semi-xenotransplantation: the regenerative medicine based-approach to immunosuppression-free transplantation and to meet the organ demand. Xenotransplantation 2014, July 8, ahead of print, with permission).

the need for a new, potentially inexhaustible source of organs and immunosuppression-free transplantation. In fact, more than 200 patients have now received body parts that were engineered from the patient's own cells, with no need for immunosuppression at any time post-surgery. At the same time, numerous labs are developing platforms and technologies aimed at the bioengineering and regeneration of transplantable organs [2]. Despite the fact that, to date, these transplanted autologous body parts have consisted of relatively simple vessels, segments of the upper airways and the urinary tract, bones, corneas, etc., the critical point is that these experiences have provided the requisite proof-of-concept for the approach. As of right now, body parts can be manufactured ex vivo. The expectation is that 'scaling up' towards more complex organs like the kidney, the heart, or the liver is inevitable, where the time-to-success will be determined largely by the will of the transplant community to invest in this field of health sciences.

Importantly, regenerative medicine has been a small research niche until the end of the first decade of this millennium, at which time the number of research groups involved in this new gold rush has started to increase exponentially. It is now time for the transplant community to realize that no other field has the potential to revolutionize transplantation more than regenerative medicine, and that - as a corollary - no other field has a larger stake in investing in regenerative medicine than organ transplantation. "The future is ours", as long as we understand that regenerative medicine is the playground where the future of organ transplantation is taking shape, and that it is time for the field to invest heavily in regenerative medicine-based approaches [3].

\section{References}

1. Salvatori M, Peloso A, Katari R, Soker S, Lerut JP, et al. (2014) Semixenotransplantation: the regenerative medicine based-approach to immunosuppression-free transplantation and to meet the organ demand. Xenotransplantation.

2. Orlando G, Soker S, Stratta RJ (2013) Organ bioengineering and regeneration as the new Holy Grail of organ transplantation. Ann Surg 258: 221-232.

3. Katari R, Peloso A, Zambon JP, Orlando G (2013) Sisyphus, the Giffen's Paradox and the Holy Grail: Time for organ transplantation to transition towards a regenerative medicine-focused type of research. Exp Rev Clin Immunol 9: 883-885. 